\title{
Association between baseline insulin resistance and psoriasis incidence: the Women's Health Initiative
}

\author{
Alfred A. Chan ${ }^{1} \cdot$ Houmin $\mathrm{Li}^{2} \cdot$ Wendy $\mathrm{Li}^{3} \cdot$ Kathy Pan ${ }^{4} \cdot$ Jennifer K. Yee ${ }^{5,6} \cdot$ Rowan T. Chlebowski ${ }^{4} \cdot$ Delphine J. Lee ${ }^{1,3,6}$
}

Received: 10 June 2021 / Accepted: 26 October 2021 / Published online: 24 November 2021

(c) The Author(s) 2021

\begin{abstract}
Small-scale studies offer conflicting evidence regarding the relationship/association between psoriasis and insulin resistance by HOMA-IR (homeostasis model assessment of insulin resistance). The purpose of this study was to assess the association between baseline HOMA-IR and psoriasis incidence in a large-scale longitudinal cohort of postmenopausal women. The analysis included 21,789 postmenopausal women from the Women's Health Initiative. Psoriasis diagnosis was defined by fee-for-service Medicare ICD-9-CM codes assigned by dermatologists or rheumatologists, and a 2-year lookback period to exclude prevalent cases. Baseline HOMA-IR was calculated using the updated HOMA2 model. Hazard rates from the Cox regression models were stratified by age (10-year intervals), on WHI component (Clinical Trial or Observational Study), and on randomization status within each of the WHI clinical trials. The complete model also adjusted for ethnicity, waist-hipratio, and smoking and alcohol habits. Among participants free of psoriasis at entry, those with high baseline HOMA-IR $(\geq 2)$ compared to low $(<1.4)$ had significantly higher risk for psoriasis over 21-year cumulative follow-up (HR: 1.39, 95\% CI 1.08-1.79, $P$-trend: 0.011). In postmenopausal women, higher baseline HOMA-IR levels were significantly associated with higher incidence of psoriasis over 21-year cumulative follow-up. Results from this time-to-event analysis indicate that insulin resistance can precede and is associated with an increased risk of psoriasis. Study is limited by Medicare diagnostic code accuracy and cohort age.
\end{abstract}

Keywords Psoriasis · Insulin resistance

\section{Introduction}

Psoriasis is a chronic inflammatory skin disease that affects about $2-4 \%$ of the U.S. population. Psoriasis can occur at any age, but peaks between age 20 and 30 years and between 50 and 60 years [1]. In postmenopausal women, the fall in estrogen concentration has been attributed to exacerbation of psoriasis [2]. According to a survey initiated by the National Psoriasis Foundation, 94\% of patients reported that psoriasis interferes with their quality of life on a daily basis [3]. Although psoriasis has been traditionally regarded as a disease limited to the skin, it is now well known that it has important health implications beyond the skin [4].

Psoriasis is associated with significant comorbidities including type 2 diabetes [5]. The current evidence hints at a complex relationship between psoriasis and insulin resistance. Small case-control studies $(n<200$ participants) have attempted to characterize the relationship between psoriasis and insulin resistance using the homeostasis model assessment of insulin resistance (HOMA-IR), 
a reliable and validated surrogate marker of insulin resistance [6-8]. However, the results have been conflicting. For example, Dhara et al. reported significantly higher HOMA-IR in psoriasis patients compared to age and sex-matched controls [9]. While Pereira et al. found that psoriasis patients are more than twice as likely to exhibit impaired glucose metabolism than controls, HOMA-IR did not significantly differ between the two groups in those with normal glucose tolerance [10]. Gyldenløve reported a significant association between psoriasis and insulin resistance when assessing with the hyperinsulinemic euglycemic clamp (HEC), but not with HOMA-IR [11].

We propose to better characterize the relationship between psoriasis and insulin resistance (via HOMA-IR) in a large cohort of postmenopausal women. Previous case-control studies have reported increased incidence of diabetes in psoriasis patients $[12,13]$. Our study instead aims to explore the reverse: whether a pre-diabetic condition such as high baseline insulin resistance is a predictor of psoriasis. To our knowledge, this is the first study using a large-scale longitudinal cohort to investigate the association between baseline insulin resistance and psoriasis incidence in postmenopausal women.

\section{Patients and methods}

\section{Study population}

The Women's Health Initiative (WHI) recruited postmenopausal women ages 50-79 years from across 40 US clinical centers between 1993 and 1998. This includes a "Clinical Trial" cohort $(n=68,132)$ with the following components: Estrogen-alone trial, Estrogen-plus-Progestin trial, Dietary Modification trial, and Calcium and Vitamin D trial. Each randomized controlled trial has its own exclusionary criteria involving safety, adherence, and retention concerns. Women ineligible or unwilling to join the clinical trials were invited to join the "Observational Study" cohort $(n=93,676)$. Detailed eligibility criteria and recruitment methods have been previously published [14]. Human subjects review committees at all participating sites approved WHI protocols and participants provided written informed consent.

Of the starting 161,808 postmenopausal women, 31,897 participants had at least one blood draw at enrollment measuring both insulin and glucose for HOMA-IR calculations. Of those, 23,093 women were linked to Medicare fee-forservice Parts A and B (FFS A + B). The final analytic cohort $(n=21,789)$ excluded 1,304 women who had prevalent cases of psoriasis or were not followed long enough for a 2-year lookback period.

\section{Data collection}

At baseline, information on demographics, medical history, and lifestyle behaviors (such as smoking, alcohol, and exercise habits) were obtained through a self-administered questionnaire. Data on lifetime hormone use were obtained by a trained interviewer, assisted by charts displaying colored photographs of various hormone preparations. Trained staff also obtained anthropometric measurements such as height, weight, and waist and hip circumferences. The total Metabolic Equivalent of Task (MET-hours per week) was calculated by multiplying the MET levels for activity by the hours exercised per week and summing the values for all activities.

\section{Psoriasis outcome ascertainment}

We classified subjects with psoriasis as previously described [15]. Briefly, psoriasis was defined by fee-forservice Medicare claims using the International Classification of Diseases, Ninth Revision, Clinical Modification (ICD-9-CM) diagnosis codes 696.0 (psoriatic arthropathy) and 696.1 (other psoriasis). To increase the validity of identifying individuals with psoriatic disease, the designation was limited to ICD-9-CM codes given by a dermatologist or rheumatologist. In a study of a managed care patient population in Northern California, psoriasis ICD-9-CM codes reported specifically by a dermatologist have a positive predictive value of $89 \%$ (95\% CI, 79-95\%) [16]. In addition, a 2-year lookback or washout period was implemented so as to not misclassify prevalent psoriasis cases as incidence [15].

\section{Determination of HOMA-IR}

Baseline blood draws (Year 0) were excluded if they were drawn after less than $12 \mathrm{~h}$ of fasting. Glucose was analyzed using the hexokinase method. Fasting insulin was analyzed by the following methods and detection systems: BMD ES3000 Immunoassay System, Roche 2010 Electrochemiluminescence, Radioimmunoassay (linco Research, St. Louis, MO), and Sandwich Immunoassay (Roche Diagnostics). The analytes were similarly distributed across the various testing methods; much of the differences could be attributed to the demographics selected for the ancillary studies (Figure S1). Insulin resistance was calculated using the HOMA2 version 2.2.3, which is an updated HOMA computer model with nonlinear solutions that account for both circulating proinsulin and variations in hepatic and peripheral glucose resistance; acceptable steady-state 
input values were 20 to $400 \mathrm{pmol} / \mathrm{L}$ for insulin and 3.0 to $25.0 \mathrm{mmol} / \mathrm{L}$ for glucose [17]. Degree of insulin resistance was categorized as defined by previous studies: Low (HOMA-IR $<1.4)$, Moderate $(1.4 \leq$ HOMA-IR $<2.0)$, and High (HOMA-IR $\geq 2.0)[18-20]$.

\section{Statistical analysis}

For primary analyses, we used time-to-event Cox proportional hazards regressions to estimate hazard ratios (HRs) and $95 \%$ confidence intervals (CIs) to study the association between baseline HOMA-IR and psoriasis incidence over the cumulative 21-year follow-up. Participants entered the risk set upon completion of the 2-year lookback period and a delayed-entry was applied for those who entered the risk set after WHI randomization. Event times were censored at the date of first psoriasis diagnosis, date no longer enrolled in FFS A + B, death or date of last follow-up through June 2017, whichever came first.

Hazard rates during follow-up were stratified on age (10-year intervals), on WHI component (Clinical Trial or Observational Study), and on randomization status within each of the WHI clinical trials (Diet Modification, Hormone Therapy, Calcium and Vitamin-D Trial). In addition, the following baseline characteristics were included in the Cox regression model to control for potential confounding effects for psoriasis based on previous literature and based on our univariable analyses: ethnicity (Caucasian, Asian, African-American, Hispanic, Other/Unspecified), continuous waist-hip-ratios, smoking (non-smoker, past-smoker, current-smoker), and alcohol habits (non-drinker, pastdrinker, current drinker) [21, 22] (Table 2). Separate models with and without adjustment for these covariates were developed and compared. The proportional hazards assumption was tested with Schoenfeld residuals, and no violation of the proportionality assumption was found. In secondary analyses, we tested for interaction between baseline HOMAIR and smoking habit on psoriasis.

\section{Results}

HOMA-IR showed a weak positive correlation with baseline BMI $(R=0.46)$ and WHR $(R=0.34)$. Figure $\mathrm{S} 2$ shows a subgroup of women who were obese (BMI $>30 \mathrm{~kg} / \mathrm{m}^{2}$ or WHR $>0.85$ ), but had low insulin resistance and vice versa. Baseline characteristics by HOMA-IR are described in Table 1. Women with high HOMA-IR $(\geq 2.0)$ were more likely to be randomized into the WHI Dietary Modification trial, but less likely to the Estrogen-plus-Progestin trial. Those with high HOMA-IR were also more likely to be younger (50-59 years), African-American, obese, prior alcohol drinkers, prior smokers, with prior hysterectomy, with fewer years of education, or fewer hours of physical activity. They were more likely to have a history of diabetes, hypertension, cardiovascular disease, stroke, liver disease, or rheumatoid arthritis. On the other hand, low HOMA-IR was associated with non-melanoma skin cancer and with the use of estrogen or estrogen-plus-progestin at baseline.

Psoriasis incidence rate was 2.36 cases per 1000 persons per year over the median cumulative follow-up of 9.5 years (interquartile range, 4.5-14.7 years). The majority of the study population was Caucasian (58.6\%) or African-American $(28.0 \%)$ and between $60-69$ years old $(47.1 \%)$. Baseline characteristics by psoriasis incidence are described in Table 2. The average age at first incidence of psoriasis was 74.6 years, occurring on average 8.3 years into the study enrollment.

The multivariable analysis adjusts for ethnicity, continuous waist-hip-ratio, smoking and alcohol habits, and was stratified on age (10-year interval), on WHI components (Clinical Trial or Observational Study), and on randomization status within each of the WHI clinical trials (Diet Modification, Hormone Therapy, Calcium and Vitamin-D Trial). Comparing psoriasis risk in HOMA-IR high $(\geq 2.0)$ versus low $(<1.4)$, the estimated hazard ratio was $1.39(95 \%$ CI 1.08-1.79, $P$-trend: 0.011) (Fig. 1). Spline-based partial hazard estimates for psoriasis was mostly linear for continuous values of HOMA-IR ( $P$-value Linear: 0.016) (Fig. 2).

In analyses stratified by smoking (a major confounder for psoriasis), no interaction between smoking and HOMAIR was detected $(P$-interaction $=0.472)$. The Kaplan-Meier curve (cumulative hazard over time) shows that among non-smoking women, the risk for psoriasis was steadily and consistently higher in women with high HOMA-IR $(\geq 2.0)$ compared to low $(<1.4)$ over the 21-year cumulative followup period (Fig. 3).

In sensitivity analyses excluding women with potentially confounding factors [such as baseline-treated diabetes $(n=1968)$, a history of hypertension $(n=8996)$, cardiovascular disease $(n=3586)$, rheumatoid arthritis $(n=1291)$, or non-melanoma skin cancer $(n=1645)]$, higher insulin resistance remained significantly associated with greater psoriasis incidence.

\section{Discussion}

Higher baseline insulin resistance assessed by the updated HOMA2 version 2.2.3 was significantly associated with greater incidence of psoriasis in postmenopausal women. Therefore, while other epidemiological studies suggest that psoriasis precedes type II diabetes [12, 13], the risk of psoriasis itself may be attributed to a pre-diabetic condition, with high insulin resistance assessed using HOMA-IR. While the exact mechanisms remain unclear, several theories 
Table 1 Baseline characteristics by HOMA-IR

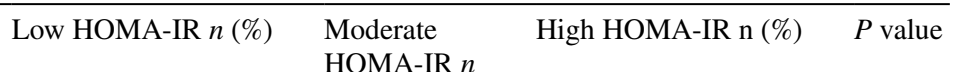
(\%)

Clinical trials

Estrogen-Alone Trial

$\begin{array}{ll} & \text { Not randomized } \\ & \text { Placebo } \\ \text { Treated }\end{array}$

Estrogen + Progestin Trial

Not randomized

Placebo

Treated

Calcium Vitamin D Trial

Not randomized

Placebo

Treated

Dietary Modification Trial

Not randomized
Placebo
Treated

Demographics

Age

$50-59$
$60-69$
$70-79$
Caucasian
Asian
African-American
Hispanic
Other/unspecified

$3601(62.8 \%)$

$6707(65.4 \%)$

$4176(72.0 \%)$

Ethnicity

Education

Type of Job

Less than high school
College
Higher

Managerial/professional
Technical/sales/admin
Service/labor
Homemaker only

Lifestyle Habits

Alcohol

\section{Never \\ Past drinker}

Current drinker

$1837(61.0 \%)$

$2795(56.6 \%)$

$9721(71.3 \%)$

Smoking

\begin{tabular}{llrrr} 
& Never & $7554(67.1 \%)$ & $1919(17.0 \%)$ & $1792(15.9 \%)$ \\
& Past smoker & $5562(65.2 \%)$ & $1476(17.3 \%)$ & $1487(17.4 \%)$ \\
& Current smoker & $1166(68.6 \%)$ & $259(15.2 \%)$ & $274(16.1 \%)$ \\
$\begin{array}{l}\text { Recreation Physical Activity } \\
\text { (MET-hour) }\end{array}$ & $\geq 0$ to $<2$ & $848(62.6 \%)$ & $225(16.6 \%)$ & $281(20.8 \%)$ \\
\hline
\end{tabular}


Table 1 (continued)

\begin{tabular}{|c|c|c|c|c|c|}
\hline & & Low HOMA-IR $n(\%)$ & $\begin{array}{l}\text { Moderate } \\
\text { HOMA-IR } n \\
(\%)\end{array}$ & High HOMA-IR n (\%) & $P$ value \\
\hline & $\geq 2$ to $<8$ & $702(59.8 \%)$ & $223(19.0 \%)$ & $249(21.2 \%)$ & \\
\hline & $\geq 8$ to $<18$ & $733(67.6 \%)$ & $187(17.3 \%)$ & $164(15.1 \%)$ & \\
\hline & $\geq 18$ & $3017(76.0 \%)$ & $509(12.8 \%)$ & $444(11.2 \%)$ & \\
\hline \multicolumn{6}{|l|}{ Anthropometric Measures } \\
\hline \multirow{4}{*}{$\begin{array}{l}\text { Baseline Body Mass Index } \\
\left(\mathrm{kg} / \mathrm{m}^{2}\right)\end{array}$} & & & & & $<0.001$ \\
\hline & Normal (18.5-24.9) & $4379(91.7 \%)$ & $288(6.0 \%)$ & $108(2.3 \%)$ & \\
\hline & Overweight (25.0-29.9) & $5618(75.1 \%)$ & $1126(15.1 \%)$ & $735(9.8 \%)$ & \\
\hline & Obese $(\geq 30.0)$ & $3847(44.5 \%)$ & $2166(25.1 \%)$ & $2628(30.4 \%)$ & \\
\hline \multirow[t]{4}{*}{ Baseline Waist to Hip Ratio } & & & & & $<0.001$ \\
\hline & Normal $(<0.800)$ & $6869(84.1 \%)$ & $798(9.8 \%)$ & $498(6.1 \%)$ & \\
\hline & Overweight (0.800-0.849) & $3825(66.9 \%)$ & $1068(18.7 \%)$ & $825(14.4 \%)$ & \\
\hline & Obese $(\geq 0.850)$ & $3574(47.1 \%)$ & $1792(23.6 \%)$ & $2223(29.3 \%)$ & \\
\hline \multicolumn{6}{|l|}{ Hormone-Related Factors } \\
\hline \multirow[t]{4}{*}{ Estrogen-alone } & & & & & $<0.001$ \\
\hline & Never & $9915(66.1 \%)$ & $2541(16.9 \%)$ & $2540(16.9 \%)$ & \\
\hline & Past & $2264(64.0 \%)$ & $658(18.6 \%)$ & $615(17.4 \%)$ & \\
\hline & Current & $2295(70.8 \%)$ & $502(15.5 \%)$ & $445(13.7 \%)$ & \\
\hline \multirow[t]{4}{*}{ Estrogen plus Progestin } & & & & & $<0.001$ \\
\hline & Never & $11,966(64.8 \%)$ & $3251(17.6 \%)$ & $3250(17.6 \%)$ & \\
\hline & Past & $1051(71.8 \%)$ & $215(14.7 \%)$ & $198(13.5 \%)$ & \\
\hline & Current & $1461(78.9 \%)$ & $235(12.7 \%)$ & $156(8.4 \%)$ & \\
\hline \multirow[t]{4}{*}{ Menstrual Cycle Regularity } & & & & & $<0.001$ \\
\hline & No & $990(62.5 \%)$ & $272(17.2 \%)$ & $321(20.3 \%)$ & \\
\hline & Yes & $12,069(66.9 \%)$ & $3068(17.0 \%)$ & $2898(16.1 \%)$ & \\
\hline & Sometimes irregular & $1306(65.4 \%)$ & $335(16.8 \%)$ & $355(17.8 \%)$ & \\
\hline \multirow[t]{5}{*}{ Age at Menopause } & & & & & $<0.001$ \\
\hline & 45 or younger & $4021(62.6 \%)$ & $1154(18.0 \%)$ & $1252(19.5 \%)$ & \\
\hline & 46 to 49 & $4419(69.4 \%)$ & $1001(15.7 \%)$ & $948(14.9 \%)$ & \\
\hline & 50 to 51 & $1837(70.0 \%)$ & $432(16.5 \%)$ & $356(13.6 \%)$ & \\
\hline & 52 or older & $3067(68.7 \%)$ & $743(16.6 \%)$ & $655(14.7 \%)$ & \\
\hline \multirow[t]{7}{*}{ Parity } & & & & & $<0.001$ \\
\hline & Never pregnant & $1403(65.5 \%)$ & $371(17.3 \%)$ & $367(17.1 \%)$ & \\
\hline & 1 & $3279(68.6 \%)$ & $794(16.6 \%)$ & $709(14.8 \%)$ & \\
\hline & 2 & $3339(68.3 \%)$ & $806(16.5 \%)$ & $746(15.3 \%)$ & \\
\hline & 3 & $2182(65.3 \%)$ & $575(17.2 \%)$ & $586(17.5 \%)$ & \\
\hline & 4 & $2439(60.9 \%)$ & $746(18.6 \%)$ & $823(20.5 \%)$ & \\
\hline & 5 & $1737(70.7 \%)$ & $376(15.3 \%)$ & $344(14.0 \%)$ & \\
\hline \multirow[t]{3}{*}{ Hysterectomy } & & & & & $<0.001$ \\
\hline & No & $8367(69.9 \%)$ & $1906(15.9 \%)$ & $1695(14.2 \%)$ & \\
\hline & Yes & $6112(62.3 \%)$ & $1793(18.3 \%)$ & $1908(19.4 \%)$ & \\
\hline \multicolumn{6}{|l|}{ Medical History } \\
\hline \multirow[t]{3}{*}{ Diabetes } & & & & & $<0.001$ \\
\hline & No & $13,825(69.8 \%)$ & $3280(16.6 \%)$ & $2698(13.6 \%)$ & \\
\hline & Yes & $650(33.0 \%)$ & $419(21.3 \%)$ & $899(45.7 \%)$ & \\
\hline \multirow[t]{3}{*}{ Hypertension } & & & & & $<0.001$ \\
\hline & No & $9365(74.5 \%)$ & $1787(14.2 \%)$ & $1422(11.3 \%)$ & \\
\hline & Yes & $4990(55.5 \%)$ & $1882(20.9 \%)$ & $2124(23.6 \%)$ & \\
\hline Cardiovascular Disease & & & & & $<0.001$ \\
\hline
\end{tabular}


Table 1 (continued)

\begin{tabular}{|c|c|c|c|c|c|}
\hline & & Low HOMA-IR $n(\%)$ & $\begin{array}{l}\text { Moderate } \\
\text { HOMA-IR } n \\
(\%)\end{array}$ & High HOMA-IR n (\%) & $P$ value \\
\hline & No & $11,382(67.7 \%)$ & $2810(16.7 \%)$ & $2617(15.6 \%)$ & \\
\hline & Yes & $2179(60.8 \%)$ & $667(18.6 \%)$ & $740(20.6 \%)$ & \\
\hline \multirow[t]{3}{*}{ Stroke } & & & & & $<0.001$ \\
\hline & No & $14,316(66.7 \%)$ & $3628(16.9 \%)$ & $3512(16.4 \%)$ & \\
\hline & Yes & $164(49.8 \%)$ & $73(22.2 \%)$ & $92(28.0 \%)$ & \\
\hline \multirow[t]{3}{*}{ Liver Disease Ever } & & & & & 0.008 \\
\hline & No & $14,187(66.6 \%)$ & $3610(17.0 \%)$ & $3500(16.4 \%)$ & \\
\hline & Yes & $296(60.4 \%)$ & $91(18.6 \%)$ & $103(21.0 \%)$ & \\
\hline \multirow[t]{3}{*}{ Bleeding Problems Ever } & & & & & 0.504 \\
\hline & No & $14,142(66.5 \%)$ & $3611(17.0 \%)$ & $3509(16.5 \%)$ & \\
\hline & Yes & $330(64.5 \%)$ & $88(17.2 \%)$ & $94(18.4 \%)$ & \\
\hline \multirow[t]{3}{*}{ History Skin Cancer } & & & & & $<0.001$ \\
\hline & No & $13,229(65.8 \%)$ & $3474(17.3 \%)$ & $3412(17.0 \%)$ & \\
\hline & Yes & $1230(74.8 \%)$ & $226(13.7 \%)$ & $189(11.5 \%)$ & \\
\hline \multirow[t]{3}{*}{ Rheumatoid Arthritis Ever } & & & & & 0.010 \\
\hline & Other/do not know & $5874(64.2 \%)$ & $1638(17.9 \%)$ & $1634(17.9 \%)$ & \\
\hline & Yes & $778(60.3 \%)$ & $242(18.7 \%)$ & $271(21.0 \%)$ & \\
\hline
\end{tabular}

The time-to-event analytic cohort consisted of 21,789 postmenopausal women. The table shows their baseline demographics, personal habits, and medical history by baseline HOMA-IR. The degree of insulin resistance was categorized as defined by previous studies: Low (HOMA-IR $<1.4)$, Moderate $(1.4 \leq$ HOMA-IR $<2.0)$, and High (HOMA-IR $\geq 2.0)(18-20)$. Differences in baseline characteristics among HOMA-IR categories were assessed using chi-square tests

$\mathrm{CI}$, confidence interval; HR, hazard ratio; MET, metabolic equivalent of task; HOMA-IR, homeostasis model assessment for insulin resistance

have been proposed for the pathophysiologic link between psoriasis and insulin resistance. This may be in part due to shared genetic regions of susceptibility between psoriasis and diabetes [23].

While the exact mechanisms that link psoriasis and insulin resistance have not been described, immune dysregulation has been reported to play a key role in both conditions [24]. T-helper cell 1 (Th1) signaling pathway have been implicated in both psoriasis and insulin resistance. A shift in the macrophage population towards more pro-inflammatory M1 than anti-inflammatory M2 has been implicated in both conditions [25]. Cytokine production through promotion of $\mathrm{NF}-\kappa \mathrm{B}$ (nuclear factor kappa light chain enhancer of activated $B$ cells) transcription factor signaling, which may also contribute to macrophage activation, has also been implicated in both conditions [26, 27]. Overproduction of proinflammatory cytokines such as tumor necrosis factor alpha $(\mathrm{TNF} \alpha)$ and interleukin (IL)-1 $\beta$ is associated with insulin resistance [28-31]. IL-1 $\beta$ blocks insulin-dependent differentiation of keratinocytes and drives keratinocyte proliferation, both of which are hallmarks of psoriasis pathogenesis [32]. $\mathrm{TNF} \alpha$ is also directly implicated in psoriasis pathogenesis and TNF $\alpha$ inhibitors are recommended as a monotherapy treatment option for adults with moderate-to-severe psoriasis [4]. These findings, combined with results from the time-to-event analysis, support the notion that insulin resistance is associated with an increased risk of psoriasis and can precede skin pathology.

Previous studies have also identified a possible relationship between psoriasis and metabolic syndrome, which is associated with insulin resistance and psoriasis severity. For example, patients with metabolic syndrome had significantly higher Psoriasis Area Severity Index (PASI) scores compared to those without metabolic syndrome. Furthermore, in patients without metabolic syndrome, HOMA-IR significantly correlated with PASI score [33]. This is also consistent with a large meta-analysis of observational studies in which patients with more severe psoriasis had greater odds of metabolic syndrome [12]. Our findings suggest that insulin resistance might be explored as an additional therapeutic target in psoriasis patients, especially in those with concomitant diabetes.

Anti-diabetic agents have anti-inflammatory properties in the setting of insulin resistance, obesity, and heart disease [34]. Optimistic reports (case reports, case series, and small clinical trials) suggest thiazolidinediones (TZDs) may provide clinical benefits for psoriasis through a decrease of cytokine production including TNF $\alpha$; however, TZDs have not been consistently shown to improve disease severity [35]. Metformin may promote macrophage 
Table 2 The distribution and risk of psoriasis by baseline demographic, personal habits, and medical history

\begin{tabular}{llll} 
No psoriasis & Psoriasis & HR & $P$ value \\
$n(\%)$ & $n(\%)$ & $(95 \% \mathrm{CI})$ & \\
\hline
\end{tabular}

Clinical Trials

Estrogen-Alone Trial

Estrogen + Progestin Trial

Calcium Vitamin D Trial

Dietary Modification Trial

Demographics

Age

Education

Lifestyle Habits

Type of Job

Ethnicity

Alcohol

Smoking
Recreation Physical Activity
(MET-hour)

Not randomized
Placebo
Treated

Not randomized
Placebo
Treated

Not randomized
Placebo

Not randomized
Placebo
Treated

Never

Past drinker

Current drinker

$$
\begin{array}{r}
11,549(97.9 \%) \\
2002(97.4 \%) \\
2081(97.7 \%)
\end{array}
$$

$11,549(97.9 \%)$

$2764(97.3 \%)$

$2901(97.9 \%)$

$14,046(97.7 \%)$

$3625(98.0 \%)$

$3626(97.7 \%)$

15,301 (97.7\%)

$2416(97.6 \%)$

$3580(98.1 \%)$

$$
\begin{aligned}
& 50-59 \\
& 60-69 \\
& 70-79
\end{aligned}
$$

Caucasian
Asian
African-American
Hispanic
Other/unspecified

Less than high school

College

Higher

Managerial/professional

Technical/sales/admin

Service/labor

Homemaker only

$5668(98.9 \%)$

$9972(97.2 \%)$

$5657(97.5 \%)$

$12,399(97.2 \%)$
$434(98.0 \%)$
$6021(98.6 \%)$
$1980(98.3 \%)$
$449(98.5 \%)$

$8048(98.0 \%)$

$7818(97.7 \%)$

$5304(97.5 \%)$

$7567(97.6 \%)$

$5648(98.0 \%)$

$4059(97.8 \%)$

$2076(97.5 \%)$

$2963(98.4 \%)$

$4829(97.9 \%)$

13,294 (97.5\%)

11,057 (98.2\%)

$8294(97.3 \%)$

$1653(97.3 \%)$

Current smoker

$\geq 0$ to $<2$

$\geq 2$ to $<8$

$1147(97.7 \%)$

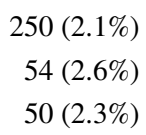

Reference

$0.79(0.56,1.10)$

0.904

0.165

$332(2.3 \%)$

$75(2.0 \%)$

$85(2.3 \%)$

Reference

1.12 (0.82, 1.53)

0.473

$365(2.3 \%)$

$59(2.4 \%)$

$68(1.9 \%)$

$-$

Reference

$0.79(0.56,1.12)$

0.190

$65(1.1 \%)$

$283(2.8 \%)$

$144(2.5 \%)$

Reference

$1.18(0.90,1.56)$

$0.84(0.62,1.13)$

0.234

0.253

$357(2.8 \%)$

$9(2.0 \%)$

$85(1.4 \%)$

$34(1.7 \%)$

$7(1.5 \%)$

Reference

$0.83(0.43,1.61)$

0.579

$0.67(0.53,0.85)$

0.001

$0.93(0.65,1.32)$

0.681

$0.76(0.36,1.60)$

0.466

\section{$166(2.0 \%) \quad$ Reference}

$184(2.3 \%)$

$1.11(0.90,1.36)$

0.346

$137(2.5 \%)$

$1.17(0.93,1.46)$

0.178

$187(2.4 \%)$

Reference

$114(2.0 \%)$

$0.84(0.67,1.06)$

0.152

$90(2.2 \%)$

$0.96(0.75,1.24)$

0.772

$54(2.5 \%)$

$1.02(0.75,1.38)$

0.910

\section{$47(1.6 \%) \quad$ Reference}

$106(2.1 \%)$

$1.45(1.03,2.04)$

0.034

$334(2.5 \%)$

$1.51(1.12,2.05)$

0.008

\section{$208(1.8 \%) \quad$ Reference}

$231(2.7 \%)$

$46(2.7 \%)$

$1.52(1.26,1.83)$

$<0.001$

$1.89(1.38,2.61) \quad<0.001$

$1310(96.8 \%) \quad 44(3.2 \%) \quad$ Reference
$27(2.3 \%)$ 
Table 2 (continued)

\begin{tabular}{|c|c|c|c|c|c|}
\hline & & $\begin{array}{l}\text { No psoriasis } \\
n(\%)\end{array}$ & $\begin{array}{l}\text { Psoriasis } \\
n(\%)\end{array}$ & $\begin{array}{l}\mathrm{HR} \\
(95 \% \mathrm{CI})\end{array}$ & $P$ value \\
\hline & $\geq 8$ to $<18$ & $1064(98.2 \%)$ & $20(1.8 \%)$ & $0.55(0.32,0.93)$ & 0.026 \\
\hline & $\geq 18$ & $3878(97.7 \%)$ & $92(2.3 \%)$ & $0.66(0.46,0.94)$ & 0.021 \\
\hline \multicolumn{6}{|c|}{ Anthropometric Measures } \\
\hline \multicolumn{6}{|c|}{ Baseline Body Mass Index } \\
\hline \multirow[t]{3}{*}{$(\mathrm{kg} / \mathrm{m} 2)$} & Normal (18.5-24.9) & $4663(97.7 \%)$ & $112(2.3 \%)$ & Reference & \\
\hline & Overweight (25.0-29.9) & $7321(97.9 \%)$ & $158(2.1 \%)$ & $0.94(0.74,1.20)$ & 0.606 \\
\hline & Obese $(\geq 30.0)$ & $8437(97.6 \%)$ & $204(2.4 \%)$ & $1.13(0.90,1.43)$ & 0.288 \\
\hline \multicolumn{6}{|c|}{ Baseline Waist to Hip Ratio } \\
\hline & Normal $(<0.800)$ & $7998(98.0 \%)$ & $167(2.0 \%)$ & Reference & \\
\hline & Overweight (0.800-0.849) & $5592(97.8 \%)$ & $126(2.2 \%)$ & $1.09(0.87,1.38)$ & 0.458 \\
\hline & Obese $(\geq 0.850)$ & $7395(97.4 \%)$ & $194(2.6 \%)$ & $1.28(1.04,1.57)$ & 0.020 \\
\hline \multicolumn{6}{|c|}{ Hormone-Related Factors } \\
\hline \multicolumn{6}{|c|}{ Estrogen-alone } \\
\hline & Never & $14,676(97.9 \%)$ & $320(2.1 \%)$ & Reference & \\
\hline & Past & $3443(97.3 \%)$ & $94(2.7 \%)$ & $1.16(0.92,1.46)$ & 0.200 \\
\hline & Current & $3164(97.6 \%)$ & $78(2.4 \%)$ & $1.26(0.98,1.61)$ & 0.068 \\
\hline \multicolumn{6}{|c|}{ Estrogen plus Progestin } \\
\hline & Never & $18,036(97.7 \%)$ & $431(2.3 \%)$ & Reference & \\
\hline & Past & $1433(97.9 \%)$ & $31(2.1 \%)$ & $0.96(0.67,1.38)$ & 0.828 \\
\hline & Current & $1822(98.4 \%)$ & $30(1.6 \%)$ & $0.82(0.56,1.18)$ & 0.285 \\
\hline \multicolumn{6}{|c|}{ Menstrual Cycle Regularity } \\
\hline & No & $1542(97.4 \%)$ & $41(2.6 \%)$ & Reference & \\
\hline & Yes & $17,623(97.7 \%)$ & $412(2.3 \%)$ & $0.85(0.61,1.17)$ & 0.308 \\
\hline & Sometimes irregular & $1959(98.1 \%)$ & $37(1.9 \%)$ & $0.70(0.45,1.09)$ & 0.116 \\
\hline \multicolumn{6}{|c|}{ Age at Menopause } \\
\hline & 45 or younger & $6289(97.9 \%)$ & $138(2.1 \%)$ & Reference & \\
\hline & 46 to 49 & $6229(97.8 \%)$ & $139(2.2 \%)$ & $1.01(0.80,1.28)$ & 0.930 \\
\hline & 50 to 51 & $2562(97.6 \%)$ & $63(2.4 \%)$ & $1.07(0.79,1.44)$ & 0.666 \\
\hline & 52 or older & $4358(97.6 \%)$ & $107(2.4 \%)$ & $0.99(0.77,1.28)$ & 0.959 \\
\hline \multicolumn{6}{|l|}{ Parity } \\
\hline & Never pregnant & $2099(98.0 \%)$ & $42(2.0 \%)$ & Reference & \\
\hline & 1 & $4656(97.4 \%)$ & $126(2.6 \%)$ & $1.29(0.91,1.83)$ & 0.152 \\
\hline & 2 & $4783(97.8 \%)$ & $108(2.2 \%)$ & $1.04(0.73,1.49)$ & 0.830 \\
\hline & 3 & $3262(97.6 \%)$ & $81(2.4 \%)$ & $1.12(0.77,1.63)$ & 0.542 \\
\hline & 4 & $3932(98.1 \%)$ & $76(1.9 \%)$ & $0.91(0.62,1.32)$ & 0.616 \\
\hline & 5 & $2403(97.8 \%)$ & $54(2.2 \%)$ & $1.05(0.70,1.57)$ & 0.827 \\
\hline \multicolumn{6}{|c|}{ Hysterectomy } \\
\hline & No & $11,714(97.9 \%)$ & $254(2.1 \%)$ & Reference & \\
\hline & Yes & $9575(97.6 \%)$ & $238(2.4 \%)$ & $1.20(1.00,1.43)$ & 0.046 \\
\hline \multicolumn{6}{|c|}{ Medical History } \\
\hline \multicolumn{6}{|l|}{ Diabetes } \\
\hline & No & $19,354(97.7 \%)$ & $449(2.3 \%)$ & Reference & \\
\hline & Yes & $1925(97.8 \%)$ & $43(2.2 \%)$ & $1.05(0.77,1.43)$ & 0.773 \\
\hline \multicolumn{6}{|c|}{ Hypertension } \\
\hline & No & $12,279(97.7 \%)$ & $295(2.3 \%)$ & Reference & \\
\hline & Yes & $8801(97.8 \%)$ & $195(2.2 \%)$ & $0.90(0.75,1.08)$ & 0.274 \\
\hline \multicolumn{6}{|c|}{ Cardiovascular Disease } \\
\hline & No & $16,446(97.8 \%)$ & $363(2.2 \%)$ & Reference & \\
\hline & Yes & $3492(97.4 \%)$ & $94(2.6 \%)$ & $1.14(0.91,1.43)$ & 0.246 \\
\hline
\end{tabular}


Table 2 (continued)

$\begin{array}{llll}\text { No psoriasis } & \text { Psoriasis } & \text { HR } & P \text { value } \\ n(\%) & n(\%) & (95 \% \mathrm{CI}) & \end{array}$

Stroke

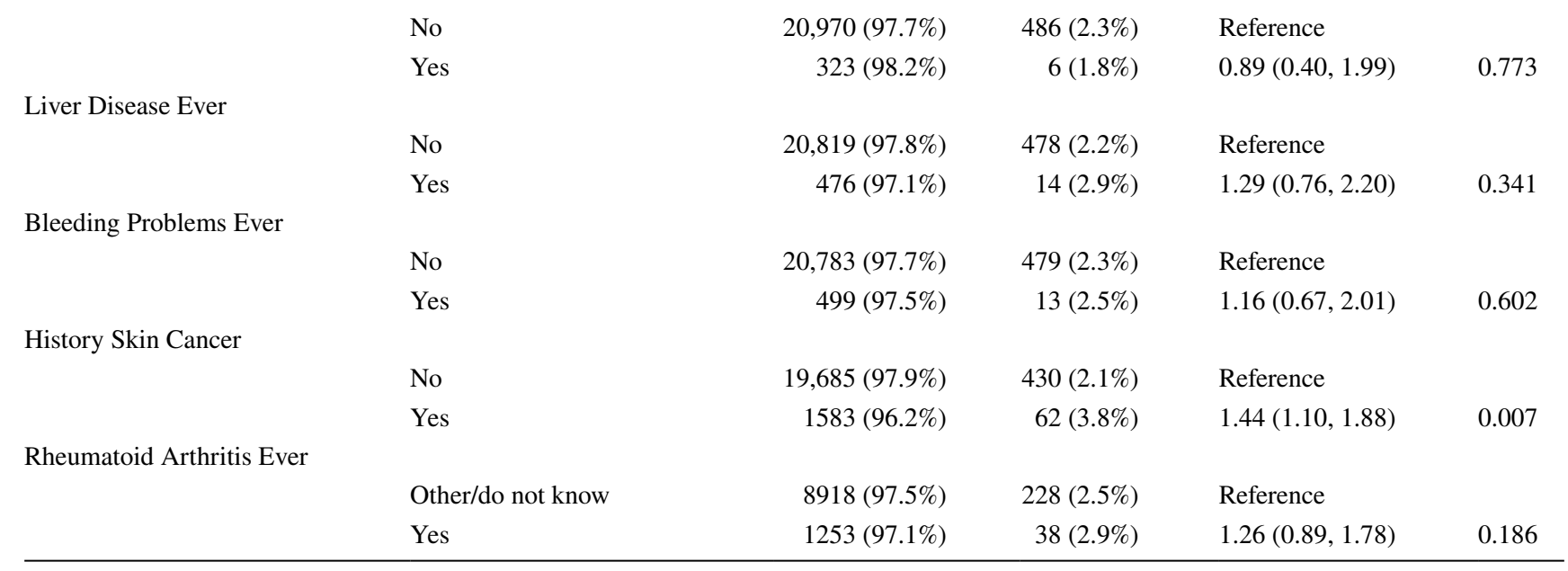

The time-to-event analytic cohort consisted of 21,789 postmenopausal women. The table shows their baseline demographics, personal habits, and medical history by psoriasis incidence over the 21 -years cumulative follow-up period. Hazard rates and $95 \%$ confidence intervals were stratified on age (10-year intervals), on WHI component (Clinical Trial or Observational Study), and on randomization status within each of the WHI clinical trials (Diet Modification, Hormone Therapy, Calcium and Vitamin-D Trial)

CI, confidence interval; HR, hazard ratio; MET, metabolic equivalent of task; HOMA-IR, homeostasis model assessment for insulin resistance

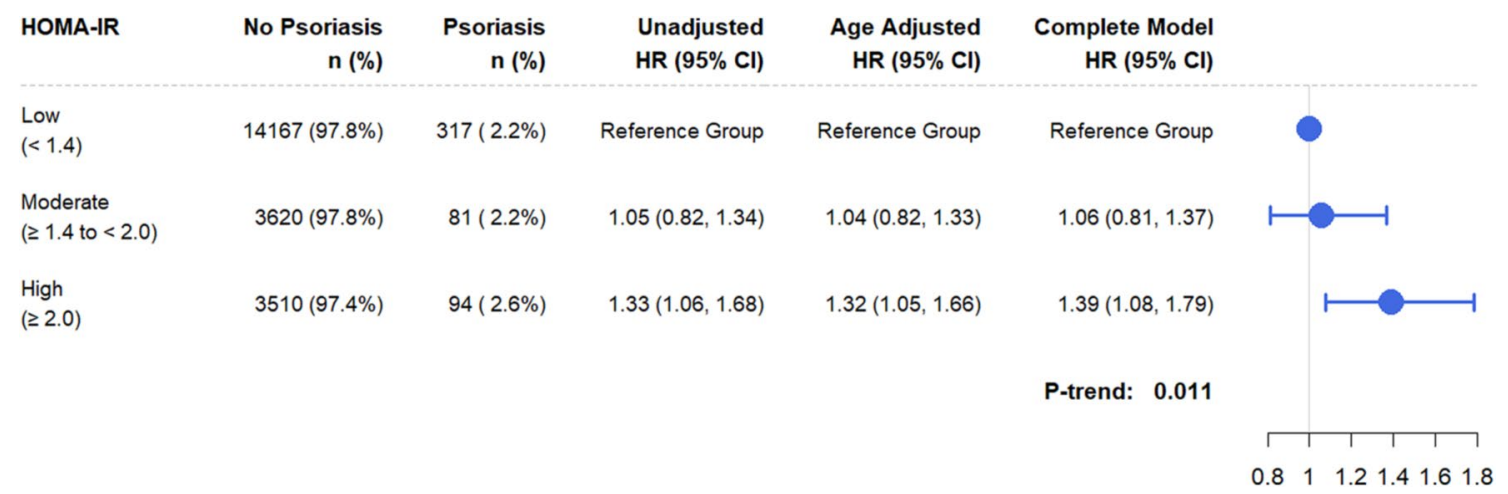

Fig. 1 Psoriasis Time-to-Event analysis by HOMA-IR. Table shows psoriasis distribution by HOMA-IR and the resulting hazard ratios (HR's) and confidence intervals (CI's) from cox-regression analysis: unadjusted, age adjusted, and the complete model. The hazard rates from the complete model were stratified on age (10-year intervals), on WHI component (Clinical or Observational Study), and on randomization status within each of the WHI clinical trials (Diet Modification, Hormone Therapy, Calcium and Vitamin-D Trial). In

activation toward the M1 phenotype through the AMPK/ $\mathrm{NF \kappa B}$ pathway [36], but other data suggest it could decrease inflammatory cytokines [37]. Human cohort data demonstrated no effect of metformin on morbidity and mortality in psoriasis patients with type 2 diabetes [38]. addition, the following baseline characteristics were included in the complete model: ethnicity (Caucasian, Asian, African-American, Hispanic, Other/Unspecified), continuous waist-hip-ratio, smoking (non-smoker, past-smoker, current-smoker), and alcohol habits (nondrinker, past-drinker, current drinker). The forest plot to the right of the table reflects the hazard ratios from the complete model and show that "high HOMA-IR" group had higher risk for psoriasis compared to the "low HOMA-IR" group

\section{Strengths and limitations}

While the accuracy of ICD-9-CM diagnostic codes has a positive predictive value of $89 \%$ (95\% CI 79-95\%) in the Northern California population, a limitation of our study is the use of ICD-9-CM diagnostic codes from Medicare 
Fig. 2 Psoriasis partial hazard (y-axis) by HOMA-IR (x-axis) as a continuous variable. The red line and the $95 \%$ confidence interval (blue shading) were predicted using a spline term on HOMA-IR with four degrees of freedom. The histogram at the bottom shows the distribution of HOMA-IR within the analytic cohort. Degree of insulin resistance was categorized as defined by previous studies: Low (HOMA-IR < 1.4), Moderate $(1.4 \leq$ HOMA-IR $<2.0)$, and High (HOMA-IR $\geq 2.0$ ) [18-20]

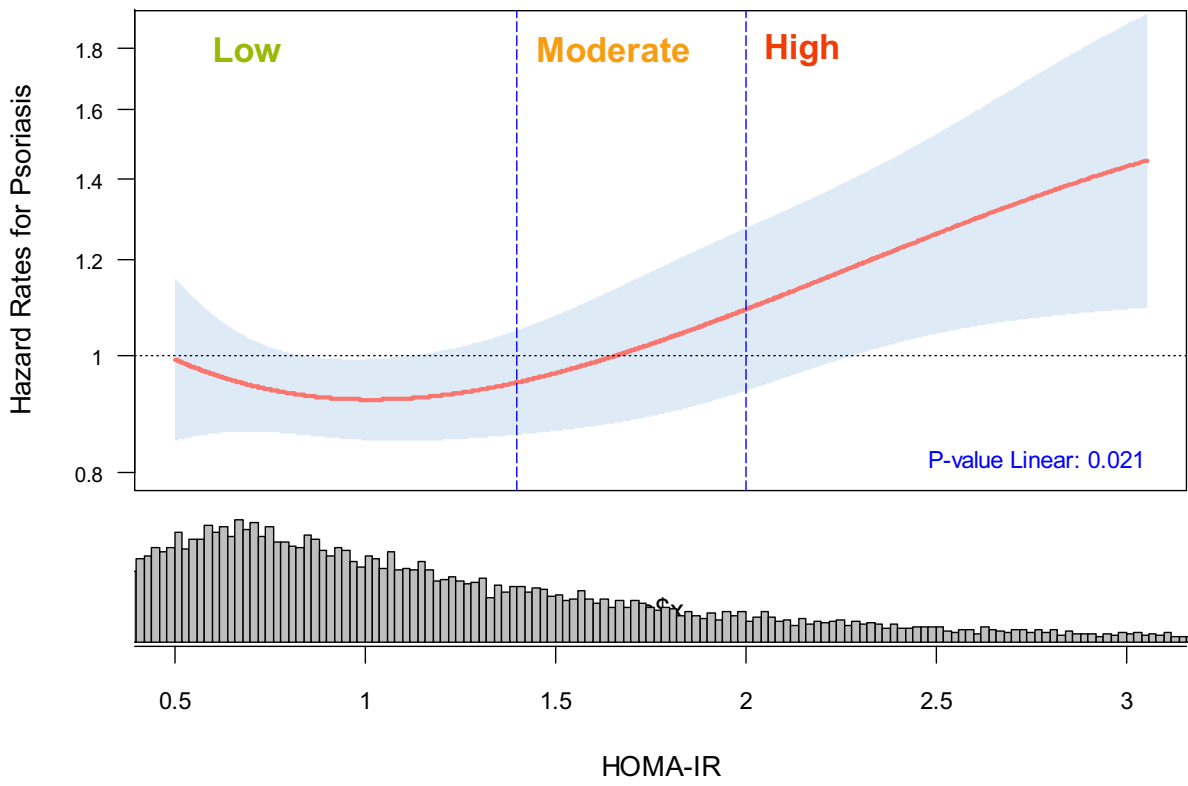

claims reports to identify psoriasis cases [16]. Although hyperinsulinemic euglycemic clamp (HEC) is considered the gold standard for evaluating insulin sensitivity, estimates derived from HOMA-IR strongly correlate with HEC [6-8, 39]. Lastly, our cohort is limited to postmenopausal women, therefore, limiting the generalizability of these findings.

Compared to previous studies (case-control studies with $n<200$ ), a key strength of our analysis is the use of a large study cohort, which provides for a more stable and robust multivariable model that accounts for key confounders for psoriasis such as obesity and smoking habits. More importantly, our analysis takes into consideration the time variable and indicates the temporal sequence between exposure and outcome compared to previous case-control studies.

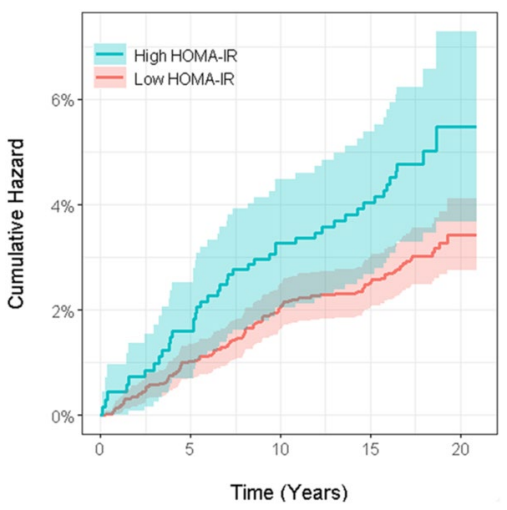

Fig. 3 Psoriasis Cumulative Hazard Curve by HOMA-IR among nonsmoking women. Over the 21-year follow-up period ( $x$-axis), the psoriasis cumulative hazard ( $y$-axis) was steadily and consistently higher in the High HOMA-IR group (Blue) compared to the Low HOMAIR group (Red) among non-smoking women. The respective colored shading represents the $95 \%$ confidence interval
Furthermore, most of the previous studies on HOMA-IR used a simple mathematical approximation while we used the updated HOMA2 version 2.2.3 [17]. Whereas BMI and WHR are subject to change in aging women, insulin resistance by HOMA-IR provides a metabolic functional measurement independent of anatomic measurements.

\section{Conclusion}

Psoriasis is a systemic inflammatory skin disease associated with significant comorbidities. In postmenopausal women, higher baseline insulin resistance assessed by HOMA-IR was significantly associated with an increased risk of psoriasis during a cumulative 21-year follow-up. While previous studies suggested that insulin resistance is a sequela of psoriasis, results from this time-to-event analysis indicate that insulin resistance can precede and is associated with an increased risk of psoriasis. Findings may warrant implementing lifestyle changes such as diet and exercise to reduce insulin resistance, which may ultimately improve or reduce the risk of psoriasis. Further research is warranted to investigate the underlying pathophysiology linking psoriasis and insulin resistance as well as the potential role of hypoglycemic agents in psoriasis management.

Supplementary Information The online version contains supplementary material available at https://doi.org/10.1007/s00403-021-02298-9.

Acknowledgements We are grateful to the volunteer subjects of the Women's Health Initiative for their participation; without them, this work would not have been possible. We also thank all the Women's Health Initiative investigators and academic centers. The WHI program is funded by the National Heart, Lung, and Blood Institute, National 
Institutes of Health, U.S. Department of Health and Human Services through contracts HHSN268201600018C, HHSN268201600001C, HHSN268201600002C, HHSN268201600003C, and HHSN268201600004C.

Author contributions AAC was involved in the data acquisition, analysis, and interpretation. HL, RTC, and DJL conceived the idea and initiated the analysis plan for the current study. AAC, HL, W.L., and DJL were involved in drafting the manuscript. RTC, JKY, and KP revised the manuscript critically for important intellectual content. All the authors approved the final version of the manuscript to be submitted for publication. DJL is the guarantor of this work and, as such, had access to data in the study and takes responsibility for integrity of the data and accuracy of the data analysis.

\section{Funding None.}

\section{Declarations}

Conflict of interest Rowan Chlebowski is a consultant for Novartis, AstraZeneca, Genentech, Amgen, Immunomedics and received honorarium from Novartis and AstraZeneca. Delphine J. Lee is a consultant for Abeona Therapeutics.

Open Access This article is licensed under a Creative Commons Attribution 4.0 International License, which permits use, sharing, adaptation, distribution and reproduction in any medium or format, as long as you give appropriate credit to the original author(s) and the source, provide a link to the Creative Commons licence, and indicate if changes were made. The images or other third party material in this article are included in the article's Creative Commons licence, unless indicated otherwise in a credit line to the material. If material is not included in the article's Creative Commons licence and your intended use is not permitted by statutory regulation or exceeds the permitted use, you will need to obtain permission directly from the copyright holder. To view a copy of this licence, visit http://creativecommons.org/licenses/by/4.0/.

\section{References}

1. Armstrong AW (2017) Psoriasis. JAMA Dermatol 153(9):956

2. Ceovic R, Mance M, Bukvic Mokos Z, Svetec M, Kostovic K, Stulhofer Buzina D (2013) Psoriasis: Female Skin Changes in Various Hormonal Stages throughout Life-Puberty, Pregnancy, and Menopause. BioMed Res Int 2013:571912

3. Armstrong AW, Schupp C, Wu J, Bebo B (2012) Quality of life and work productivity impairment among psoriasis patients: findings from the National Psoriasis Foundation survey data 20032011. PLoS ONE 7(12):e52935

4. Menter A, Strober BE, Kaplan DH, Kivelevitch D, Prater EF, Stoff B et al (2019) Joint AAD-NPF guidelines of care for the management and treatment of psoriasis with biologics. J Am Acad Dermatol 80(4):1029-1072

5. Kimball AB, Gladman D, Gelfand JM, Gordon K, Horn EJ, Korman NJ et al (2008) National Psoriasis Foundation clinical consensus on psoriasis comorbidities and recommendations for screening. J Am Acad Dermatol 58(6):1031-1042

6. Bonora E, Targher G, Alberiche M, Bonadonna RC, Saggiani F, Zenere MB et al (2000) Homeostasis model assessment closely mirrors the glucose clamp technique in the assessment of insulin sensitivity: studies in subjects with various degrees of glucose tolerance and insulin sensitivity. Diabetes Care 23(1):57-63
7. Emoto M, Nishizawa Y, Maekawa K, Hiura Y, Kanda H, Kawagishi T et al (1999) Homeostasis model assessment as a clinical index of insulin resistance in type 2 diabetic patients treated with sulfonylureas. Diabetes Care 22(5):818-822

8. Matthews DR, Hosker JP, Rudenski AS, Naylor BA, Treacher DF, Turner RC (1985) Homeostasis model assessment: insulin resistance and beta-cell function from fasting plasma glucose and insulin concentrations in man. Diabetologia 28(7):412-419

9. Dhara S, Dasgupta A, Rout JK, Banerjee U, Dasgupta S, Ghosh A (2015) Clinico-biochemical correlation between psoriasis and insulin resistance. Indian J Clin Biochem 30(1):99-103

10. Pereira RR, Amladi ST, Varthakavi PK (2011) A study of the prevalence of diabetes, insulin resistance, lipid abnormalities, and cardiovascular risk factors in patients with chronic plaque psoriasis. Indian J Dermatol 56(5):520-526

11. Gyldenlove M, Storgaard H, Holst JJ, Vilsboll T, Knop FK, Skov L (2015) Patients with psoriasis are insulin resistant. J Am Acad Dermatol 72(4):599-605

12. Armstrong AW, Harskamp CT, Armstrong EJ (2013) Psoriasis and the risk of diabetes mellitus: a systematic review and metaanalysis. JAMA Dermatol 149(1):84-91

13. Cheng J, Kuai D, Zhang L, Yang X, Qiu B (2012) Psoriasis increased the risk of diabetes: a meta-analysis. Arch Dermatol Res 304(2):119-125

14. Group TWsHI (1998). Design of the Women's Health Initiative clinical trial and observational study. The Women's Health Initiative Study Group. Control Clin Trials 19(1):61-109

15. Chan AA, Noguti J, Yang CT, Feldman SR, Wallace RB, Shadyab AH et al (2021) Association between menopausal hormone therapy and incidence of psoriasis: a secondary analysis from the women's health initiative randomized clinical trials. Br J Dermatol

16. Asgari MM, Wu JJ, Gelfand JM, Salman C, Curtis JR, Harrold LR et al (2013) Validity of diagnostic codes and prevalence of psoriasis and psoriatic arthritis in a managed care population, 1996-2009. Pharmacoepidemiol Drug Saf 22(8):842-849

17. Wallace TM, Levy JC, Matthews DR (2004) Use and abuse of HOMA modeling. Diabetes Care 27(6):1487-1495

18. Bermudez V, Rojas J, Martinez MS, Apruzzese V, Chavez-Castillo M, Gonzalez R et al (2014) Epidemiologic behavior and estimation of an optimal cut-off point for homeostasis model assessment-2 insulin resistance: a report from a Venezuelan population. Int Sch Res Notices. 2014:616271

19. Ghasemi A, Tohidi M, Derakhshan A, Hasheminia M, Azizi F, Hadaegh F (2015) Cut-off points of homeostasis model assessment of insulin resistance, beta-cell function, and fasting serum insulin to identify future type 2 diabetes: Tehran Lipid and Glucose Study. Acta Diabetol 52(5):905-915

20. Lee CH, Shih AZ, Woo YC, Fong CH, Leung OY, Janus E et al (2016) Optimal Cut-Offs of Homeostasis Model Assessment of Insulin Resistance (HOMA-IR) to Identify Dysglycemia and Type 2 Diabetes Mellitus: A 15-Year Prospective Study in Chinese. PLoS ONE 11(9):e0163424

21. Naldi L (2016) Psoriasis and smoking: links and risks. Psoriasis (Auckl) 6:65-71

22. Setty AR, Curhan G, Choi HK (2007) Smoking and the risk of psoriasis in women: Nurses' Health Study II. Am J Med 120(11):953-959

23. Wang H, Wang Z, Rani PL, Fu X, Yu W, Bao F et al (2017) Identification of PTPN22, ST6GAL1 and JAZF1 as psoriasis risk genes demonstrates shared pathogenesis between psoriasis and diabetes. Exp Dermatol 26(11):1112-1117

24. Friis NU, Hoffmann N, Gyldenlove M, Skov L, Vilsboll T, Knop FK et al (2019) Glucose metabolism in patients with psoriasis. Br J Dermatol 180(2):264-271

25. Lu CH, Lai CY, Yeh DW, Liu YL, Su YW, Hsu LC et al (2018) Involvement of M1 macrophage polarization in endosomal 
toll-like receptors activated psoriatic inflammation. Mediators Inflamm 2018:3523642

26. Olefsky JM, Glass CK (2010) Macrophages, inflammation, and insulin resistance. Annu Rev Physiol 72:219-246

27. Sun Y, Zhang J, Zhou Z, Wu P, Huo R, Wang B et al (2015) $\mathrm{CCN} 1$, a pro-inflammatory factor, aggravates psoriasis skin lesions by promoting keratinocyte activation. J Invest Dermatol 135(11):2666-2675

28. Akash MSH, Rehman K, Liaqat A (2018) Tumor Necrosis FactorAlpha: Role in Development of Insulin Resistance and Pathogenesis of Type 2 Diabetes Mellitus. J Cell Biochem 119(1):105-110

29. Hotamisligil GS, Peraldi P, Budavari A, Ellis R, White MF, Spiegelman BM (1996) IRS-1-mediated inhibition of insulin receptor tyrosine kinase activity in TNF-alpha- and obesityinduced insulin resistance. Science 271(5249):665-668

30. Jager J, Gremeaux T, Cormont M, Le Marchand-Brustel Y, Tanti JF (2007) Interleukin-1beta-induced insulin resistance in adipocytes through down-regulation of insulin receptor substrate-1 expression. Endocrinology 148(1):241-251

31. Nov O, Kohl A, Lewis EC, Bashan N, Dvir I, Ben-Shlomo S et al (2010) Interleukin-1beta may mediate insulin resistance in liverderived cells in response to adipocyte inflammation. Endocrinology 151(9):4247-4256

32. Buerger C, Richter B, Woth K, Salgo R, Malisiewicz B, Diehl S et al (2012) Interleukin-1beta interferes with epidermal homeostasis through induction of insulin resistance: implications for psoriasis pathogenesis. J Invest Dermatol 132(9):2206-2214

33. Polic MV, Miskulin M, Smolic M, Kralik K, Miskulin I, Berkovic $\mathrm{MC}$ et al (2018) Psoriasis severity-A risk factor of insulin resistance independent of metabolic syndrome. Int J Environ Res Public Health 15(7)

34. Pollack RM, Donath MY, LeRoith D, Leibowitz G (2016) Antiinflammatory agents in the treatment of diabetes and its vascular complications. Diabetes Care 39(Supplement 2):S244-S252

35. Ip W, Kirchhof MG (2017) Glycemic control in the treatment of psoriasis. Dermatology 233(1):23-29

36. Chiang CF, Chao TT, Su YF, Hsu CC, Chien CY, Chiu KC et al (2017) Metformin-treated cancer cells modulate macrophage polarization through AMPK-NF-kappaB signaling. Oncotarget 8(13):20706-20718

37. Cameron AR, Morrison VL, Levin D, Mohan M, Forteath C, Beall $\mathrm{C}$ et al (2016) Anti-inflammatory effects of metformin irrespective of diabetes status. Circ Res 119(5):652-665

38. Su Y-J, Chen T-H, Hsu C-Y, Chiu W-T, Lin Y-S, Chi C-C (2019) Safety of metformin in psoriasis patients with diabetes mellitus: a 17-year population-based real-world cohort study. J Clin Endocrinol Metab 104(8):3279-3286

39. Brenelli SL, Moraes AM, Monte-Alegre S, Carvalho OM, Saad MJ (1995) Insulin resistance in psoriasis. Br J Med Biol Res 28(3):297-301

Publisher's Note Springer Nature remains neutral with regard to jurisdictional claims in published maps and institutional affiliations. 